\title{
IWS: Integrated web server for protein sequence and structure analysis
}

\author{
Khader Shameer ${ }^{1}$ and Ramanathan Sowdhamini ${ }^{1}$ * \\ ${ }^{1}$ National Centre for Biological Sciences (TIFR), GKVK Campus, Bellary Road, Bangalore 560 065, India; \\ Ramanathan Sowdhamini* - E-mail: mini@ncbs.res.in; * Corresponding author \\ received October 24, 2007; accepted October 29, 2007; published online November 01, 2007
}

\begin{abstract}
:
Rapid increase in protein sequence information from genome sequencing projects demand the intervention of bioinformatics tools to recognize interesting gene-products and associated function. Often, multiple algorithms need to be employed to improve accuracy in predictions and several structure prediction algorithms are on the public domain. Here, we report the availability of an Integrated Web-server as a bioinformatics online package dedicated for in-silico analysis of protein sequence and structure data (IWS). IWS provides web interface to both in-house and widely accepted programs from major bioinformatics groups, organized as 10 different modules. IWS also provides interactive images for Analysis Work Flow, which will provide transparency to the user to carry out analysis by moving across modules seamlessly and to perform their predictions in a rapid manner.
\end{abstract}

Availability: IWS is available from the URL: http://caps.ncbs.res.in/iws

Key Words: protein sequence; structure analysis

Background:

Bioinformatics is now in a transition state - "from a datacentric science to knowledge based science”, analysis and extraction of relevant information from huge amount of data from various high-throughput experiments remains as the major challenge in bioinformatics. Rapid increase in data generation has left us with many genes and proteins as 'unknown' or 'hypothetical' ones. As it is impossible to validate all the sequence data by means of biochemical experiments for confirmation of the likely associations, bioinformatics approaches can play an important role as a filter for recognizing potential gene products that can represent new fold or a novel function. Computational approaches enable the recognition of putative gene products of a family and to rationally design mutation experiments. Along with rapid incoming data, the availability of various resources to analyze the data has also increased. IWS is a compilation of in-house databases, web servers and web interface for various programs related to protein sequence and structure analyses clustered as ten modules. IWS is an easy-to-use web server, which will enable the novice as well as the expert users to carry out protein sequence and structure analysis rapidly and easily.

ISSN 0973-2063

Bioinformation 2(3): 86-90 (2007)

\section{Implementation:}

IWS provides various tools and database related to protein sequence and structure analysis classified into 10 different modules. Detailed information about the available modules, various tools, URL and its applications are given in Table 1 (supplementary material). IWS provides the tools and database under the following 10 different modules: Database and Servers, Sequence Retrieval and Search, Alignment, Sequence Analysis, Secondary Structure Prediction, Structure Analysis, Protein Modeling and Structure Validation, Sequence-Structure analysis, Phylogeny and Fold Recognition. Some of the major programs and databases available from IWS are PSI-BLAST [1], CASCADE PSI-BLAST [2], PHYLIP [3], SEQPLOT, JOY [4], MODIP [5], SCANMOT [6], MODELLER [7], HARMONY [8], PASS2 [9], DSDBASE [10] etc. More than 40 bioinformatics resources for protein sequence and structure analysis is available from IWS. Figure 1 illustrates a flowchart that explains about different databases and tools available from IWS. IWS is running on a CentOS-Apache server. Front-end of IWS is developed using HTML, Perl script, CGI script, and Java scripts. Back-end is a combination of different programs developed using different languages like FORTRAN, C library (GD), C++, and Perl. 


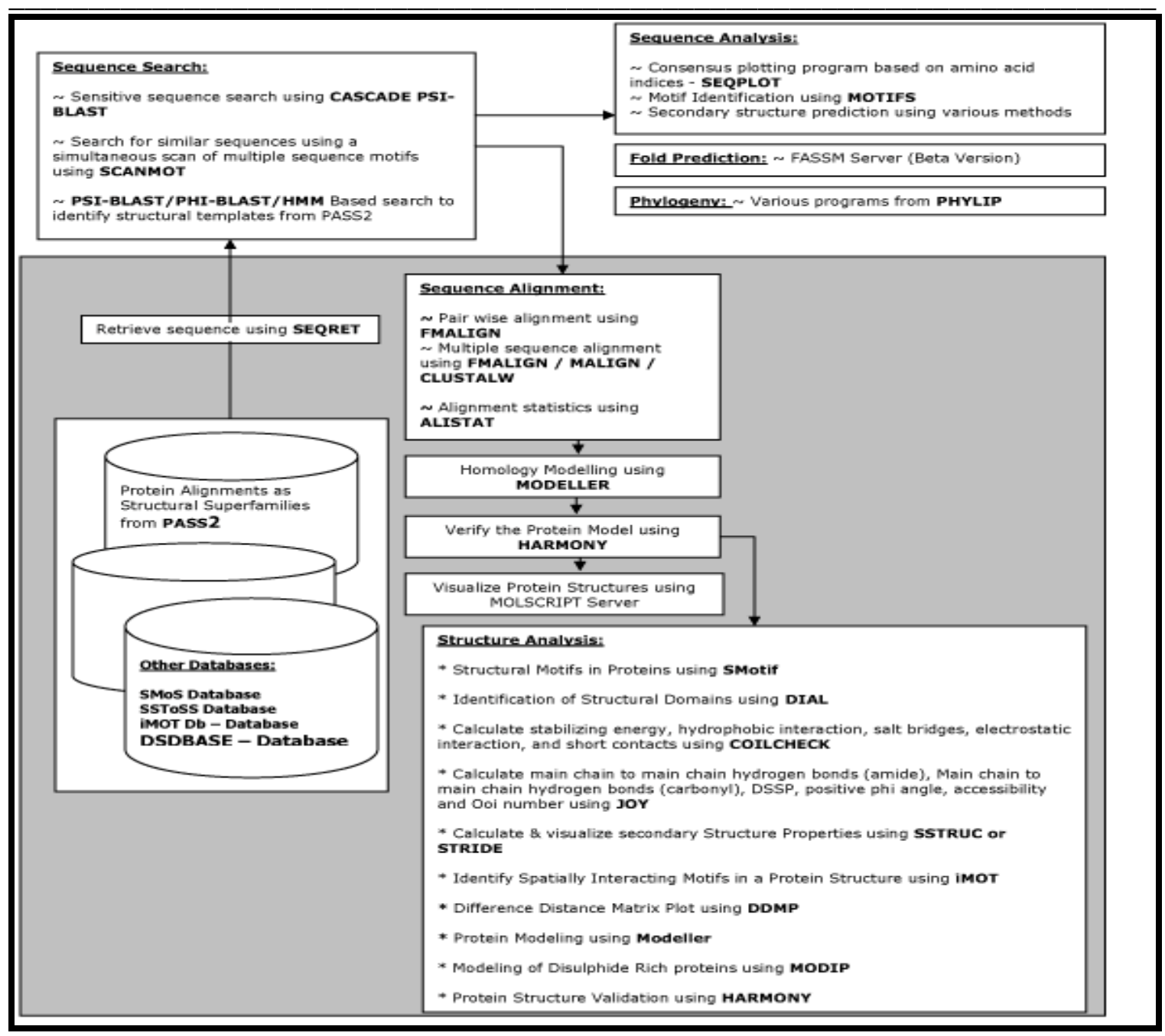

Figure 1: Schematic flow-chart about different databases and tools available from IWS

\section{Input-output options:}

IWS accepts sequences in FASTA, PIR, and Phylip format for protein sequence analysis and PDB file format for structure analysis. IWS generates different output for different programs. For instance, we have projected the results of the run on an 'unknown protein' sequence (from Drosophila ambigua (gi: 3676414) from NCBI Protein database) at the URL: http://caps.ncbs.res.in/iws/unknown/example1.html.

\section{Caveats and future directions:}

IWS can be accessible using any standard web-browsers (IE, Mozilla, Opera, Firefox etc.). We are planning to upgrade IWS version 2 as cluster-based server. We will continue to integrate new tools as they become available from the lab.

\section{Conclusion:}

In this article, we have explained about the availability of a new web server, NCBS-IWS an integrated web server ISSN 0973-2063 Bioinformation 2(3): 86-90 (2007) for protein sequence and structure analysis. IWS will be a useful resource for research and academic communities interested in protein sequence and structure analysis.

\section{Acknowledgement:}

R.S. was a Senior Research Fellow of the Wellcome Trust; U.K. K.S. was briefly supported by the Wellcome Trust Grant. We thank Mr. G. Pugalenthi for useful discussions. We also thank NCBS (TIFR) for financial and infrastructural support.

\section{References:}

[01] S. F. Altschul, et al., Nucleic Acids Res., 25: 3389 (1997) [PMID: 9254694]

[02] S. Sandhya, et al., J Biomol Struct Dyn., 3:283 (2005) [PMID: 16218755]

[03] J. Felsenstein, Annu Rev Genet., 22: 521 (1988) [PMID: 3071258]

[04] K. Mizuguchi, et al., Bioinformatics, 14: 617 (1998) [PMID: 9730927] 


\section{Bioinformation}

www.bioinformation.net

open access

[05] R. Sowdhamini, et al., Prot. Engng., 3: 95 (1989) [PMID: 2594728]

[06] S. Chakrabarti, et al., BMC Bioinformatics, 5: 167 (2004) [PMID: 15509307]

[07] A. Sali, and T. L. Blundell, J Mol Biol., 234: 779 (1993) [PMID: 8254673]
[08] G. Pugalenthi, et al., Nucleic Acids Res., 34: W231 (2006) [PMID: 16844999]

[09] A. Bhaduri, et al., BMC Bioinformatics, 5: 35 (2004) [PMID: 15059245]

[10] A. Vinayagam, et al., Nucleic Acids Res., 32: D200 (2004) [PMID: 14681394]

Edited by $P$. Kangueane

Citation: Shameer \& Sowdhamini, Bioinformation 2(3): 86-90 (2007)

License statement: This is an open-access article, which permits unrestricted use, distribution, and reproduction in any medium, for non-commercial purposes, provided the original author and source are credited. 


\section{Bioinformation}

\section{www.bioinformation.net}

\begin{tabular}{|c|c|c|c|}
\hline \multicolumn{4}{|c|}{ Supplementary material: } \\
\hline No & Tool/Database & URL & Application \\
\hline \multicolumn{4}{|c|}{ Module 1 : Database \& Server } \\
\hline 1 & Database \& Server & http://caps.ncbs.res.in/iws/dbs.html & $\begin{array}{l}\text { List of selected database and web servers related to } \\
\text { protein sequence and structure analysis. }\end{array}$ \\
\hline \multicolumn{4}{|c|}{ Module 2: Sequence Search } \\
\hline 2 & $\begin{array}{l}\text { CASCADE PSI- } \\
\text { BLAST }\end{array}$ & http://caps.ncbs.res.in/iws/cascade.html & $\begin{array}{l}\text { PSI-BLAST for many 'generations', initiating } \\
\text { searches from new homologues }\end{array}$ \\
\hline 3 & SCANMOT & http://caps.ncbs.res.in/scanmot/scanmot.html & $\begin{array}{l}\text { Search for similar sequences using a simultaneous } \\
\text { scan of multiple sequence motifs }\end{array}$ \\
\hline 4 & $\begin{array}{l}\text { PSI-BLAST Search } \\
\text { PASS2 Database }\end{array}$ & http://caps.ncbs.res.in/iws/psib_pass2.html & PSI-BLAST Search using PASS2 Database \\
\hline 5 & $\begin{array}{l}\text { PHI-BLAST Search } \\
\text { against PASS2 } \\
\text { Database }\end{array}$ & http://caps.ncbs.res.in/iws/phib_pass2.html & PHI-BLAST Search using PASS2 Database \\
\hline 6 & $\begin{array}{l}\text { HMM Search against } \\
\text { PASS2 }\end{array}$ & http://caps.ncbs.res.in/iws/hmm_pass2.html & $\begin{array}{l}\text { HMM Search against PASS2 Database to identify } \\
\text { Structural Templates }\end{array}$ \\
\hline 7 & SEQRET & http://caps.ncbs.res.in/iws/seqret.html & $\begin{array}{l}\text { Retrieve Protein Sequence from Swiss- } \\
\text { Prot/NR/TrEMBL using user Query / Accession ID }\end{array}$ \\
\hline \multicolumn{4}{|c|}{ Module 3: Alignment } \\
\hline 8 & FMALIGN & http://caps.ncbs.res.in/iws/FMAmulti.html & $\begin{array}{l}\text { Fixed Motif ALIGNment [FMALIGN] - Pair-wise } \\
\text { \& Multiple Alignment }\end{array}$ \\
\hline 9 & MALIGN & http://caps.ncbs.res.in/iws/malign.html & Multiple Sequence Alignment using MALIGN \\
\hline 10 & $\begin{array}{l}\text { MALIGN Search } \\
\text { against PASS2 }\end{array}$ & http://caps.ncbs.res.in/iws/malign_pass2.html & $\begin{array}{l}\text { MALIGN Search against PASS2 Database to } \\
\text { identify Structural templates for protein modeling }\end{array}$ \\
\hline 11 & ALISTAT & http://caps.ncbs.res.in/iws/alistat_ali.html & Alignment Statistics using ALISTAT \\
\hline 12 & $\begin{array}{l}\text { iMOT from } \\
\text { Alignments }\end{array}$ & http://caps.ncbs.res.in/iws/imot_ali.html & $\begin{array}{l}\text { Find iMOT(Interacting MOTifs) from an alignment } \\
\text { using iMOT Algorithm }\end{array}$ \\
\hline \multicolumn{4}{|c|}{ Module 4: Sequence Analysis } \\
\hline 13 & SEQPLOT & http://caps.ncbs.res.in/iws/seqplot.html & $\begin{array}{l}\text { Consensus Sequence plotting program based on } \\
\text { various amino acid indices }\end{array}$ \\
\hline 14 & DIAL Sequence & http://caps.ncbs.res.in/iws/dial_seq.html & $\begin{array}{l}\text { Identify Structural Domains from Protein Sequence } \\
\text { using DIAL (Domain Identification ALgorithm) }\end{array}$ \\
\hline 15 & $\begin{array}{l}\text { iMOT Conserved } \\
\text { Regions }\end{array}$ & http://caps.ncbs.res.in/iws/imot_cr.html & $\begin{array}{l}\text { Identify Sequentially conserved regions in Proteins } \\
\text { sequence using iMOT Algorithm }\end{array}$ \\
\hline 16 & $\begin{array}{l}\text { iMOT Spatially } \\
\text { Interacting Motifs }\end{array}$ & http://caps.ncbs.res.in/iws/imot_seq.html & $\begin{array}{l}\text { Identify Spatially Interacting Motifs in Proteins } \\
\text { sequence using iMOT Algorithm }\end{array}$ \\
\hline 17 & MOTIFS & http://caps.ncbs.res.in/iws/motifs.html & $\begin{array}{l}\text { Identify sequence motifs from Protein Sequence } \\
\text { using MOTIFS program }\end{array}$ \\
\hline 18 & SEQREPORT & http://caps.ncbs.res.in/iws/seqreport.php & $\begin{array}{l}\text { Amino acid composition, Molecular weight, Molar } \\
\text { absorption coefficient, Protein iso-electric point with } \\
\text { pK values, Charge at different } \mathrm{pH} \text {, sequence as } 3 \\
\text { letters amino acid code and Classification of amino } \\
\text { acid residues }\end{array}$ \\
\hline 19 & $\begin{array}{l}\text { Secondary Structure } \\
\text { Analysis }\end{array}$ & http://caps.ncbs.res.in/iws/secstr.html & $\begin{array}{l}\text { Access Various Secondary Structure Servers } \\
\text { through a single Window of NCBS-IWS }\end{array}$ \\
\hline \multicolumn{4}{|c|}{ Module 5: Phylogeny } \\
\hline 20 & PROTPARS & http://caps.ncbs.res.in/iws/protpars.html & $\begin{array}{l}\text { PROTPARS - Estimate phylogenies from protein } \\
\text { sequences (PHYLIP) }\end{array}$ \\
\hline 21 & PROTDIST & http://caps.ncbs.res.in/iws/protdist.html & $\begin{array}{l}\text { PROTDIST - Computes a distance measure for } \\
\text { protein sequences (PHYLIP) }\end{array}$ \\
\hline 22 & PROML & http://caps.ncbs.res.in/iws/proml.html & $\begin{array}{l}\text { PROML - Estimates phylogenies from protein } \\
\text { amino acid sequences by maximum likelihood } \\
\text { (PHYLIP) }\end{array}$ \\
\hline 23 & PROMLK & http://caps.ncbs.res.in/iws/promlk.html & $\begin{array}{l}\text { PROMLK - Same as PROML but assumes a } \\
\text { molecular clock (PHYLIP) }\end{array}$ \\
\hline 24 & SEQBOOT & http://caps.ncbs.res.in/iws/seqboot.html & $\begin{array}{l}\text { SEQBOOT - Reads in a data set, and produces } \\
\text { multiple data sets from it by bootstrap re-sampling }\end{array}$ \\
\hline
\end{tabular}


Bioinformation

www.bioinformation.net

\section{Web Server}

\author{
25 CONSENSE \\ 26 NEIGHBOR \\ $27 \quad$ KITSCH \\ Module 6: Secondary Structure Prediction \\ 28 Integrated web- \\ interface for \\ Secondary Structure \\ Prediction
}

\section{Module 7: Sequence-Structure Analysis}

29 SSTOSS Database

http://caps.ncbs.res.in/SSTOSS/passlist.htm

$30 \quad$ JOY

http://caps.ncbs.res.in/iws/joy_tem.html
http://caps.ncbs.res.in/SMotif http://caps.ncbs.res.in/iws/dial_struc.html http://caps.ncbs.res.in/iws/coilcheck.html

33 COILCHECK

34 STRIDE

SSTRUC

HBOND

C-Alpha Calculation

38 atm2seq

39 JOY Structural

features

iMOTdb

DDMP

SMoS Database http://caps.ncbs.res.in/iws/stride.html

http://caps.ncbs.res.in/iws/sstruc.html http://caps.ncbs.res.in/iws/hbond.html http://caps.ncbs.res.in/iws/cadistance.html

http://caps.ncbs.res.in/iws/atm2ali.html

http://caps.ncbs.res.in/iws/joy_tem.html

http://caps.ncbs.res.in/imotdb http://caps.ncbs.res.in/iws/ddmatrix.html http://caps.ncbs.res.in/SMoS

http://caps.ncbs.res.in/iws/molscript.html

http://caps.ncbs.res.in/iws/fassm.html

\section{Module 10: Protein Modeling \& Structure Validation}

45 MODIP

46 DSDBASE Search

http://caps.ncbs.res.in/iws/modip.html http://caps.ncbs.res.in/dsdbase//accessmts.ht $\mathrm{ml}$

47 MODELLER* (License Required) 48 HARMONY

(PHYLIP)

CONSENSE - Computes consensus trees by the majority-rule consensus tree method (PHYLIP)

NEIGHBOR - Neighbor-Joining method of Saitou and Nei (1987) and the UPGMA method of clustering (PHYLIP)

KITSCH - Fitch-Margoliash and Least Squares Methods with Evolutionary Clock (PHYLIP)

Jpred, BetaTurnv1.1 - Prediction of Beta-turn By Support Vector Machine, SPLIT - Membrane Protein Secondary Structure Prediction, HMMTOP Transmembrane Helices \& Topology, Sosui Classification and Secondary Structure Prediction of Membrane Proteins

SSToSS : A database of Sequence Structural Templates of Single member Superfamilies

Calculate main-chain to main-chain hydrogen bonds (amide), Main-chain to main-chain hydrogen bonds (carbonyl), DSSP, positive phi angle, accessibility and Ooi number using JOY Program

SMotif - Structural Motifs in Proteins

Identify Structural Domains using DIAL (Domain Identification ALgorithm)

Calculate Stabilizing Energy, Hydrophobic Interaction, Salt Bridges, Electrostatic Interaction, and Short Contacts using COILCHECK

Calculate \& Visualise Secondory Structure Properties using STRIDE

Secondory Structural Patterns using SSTRUC

Hydrogen bonding Properties using HBOND

Calculate C-alpha distance between two atoms of a PDB file

Extract Sequence Information from a PDB file using atm2seq

Generate JOY Structural features output for a PDB File

Database of Spatially Interacting Motifs in Proteins Difference Distance Matrix using DDMP

Structural Motifs of Superfamilies Database provides information on structural motifs or templates of aligned protein domain superfamilies like PASS2 and CAMPASS

Generate MOLSCRIPT Images of Macromolecules

Neural Network based FASSM (Enhanced Function Association in whole genome analysis using Sequence and Structural Motifs.) Server for fold prediction

MODIP(Modeling Of DIsulphide bonds in Proteins) Search for Disulphide rich proteins using DSDBASE Search Tool

Build Proteins using MODELLER

Validate Protein Models using HARMONY

Table 1: Detailed list of Tools with name, URL and application grouped in to 10 different modules 